\title{
Presentación
}

\section{Medios digitales, entre la disrupción y la transformación}

L

a presente edición de la revista Contratexto tiene como objetivo considerar investigaciones interdisciplinarias con énfasis en el estudio de los fenómenos generados por la disrupción y la transformación digital en los distintos espacios y aspectos de nuestras sociedades. La convocatoria a esta edición buscó seleccionar especialmente trabajos provenientes de las ciencias humanas y encontrar un balance entre la teoría y la práctica, esto más que todo para mostrar las distintas propuestas metodológicas aplicadas al estudio de los fenómenos sobre los que la web impacta. Así pues, este número da respuesta a esa creciente necesidad de canalizar y agrupar investigaciones de este tipo, poniendo a disposición un espacio donde estos trabajos puedan exponerse desde la perspectiva aquí descrita.

Más allá de lo práctico que pueda ser la publicación de un dosier de trabajos orientados a la comunicación digital, es importante sumarse a la reflexión desde la visión académica, donde a primera vista hemos podido observar cómo la World Wide Web, a través de la estructura de internet, se ha venido popularizando desde los años noventa hasta llegar a adquirir un mayor significado en los diversos ámbitos de nuestras sociedades. Es justamente este detalle el que no ha pasado inadvertido para la ciencia, tanto así que en los últimos años se ha planteado la necesidad de estudiar la web desde una disciplina propia (Berners-Lee, Hall, Hendler, Shadbolt y Weitzner, 2006). Aunque esta propuesta editorial no pretende entrar en la dis- 
cusión sobre si es necesario instalar una disciplina que se encargue de estudiar la web, y tampoco se suma a la postura que teme una atomización de las ciencias, sí se esmera en entregar estudios que giran en torno al objeto dentro de una edición que busca por primera vez considerarlas bajo un manto que las una.

En una observación más detallada, hoy a la luz de una creciente complejidad del sistema en el que se encuentra la web, con ciclos de evolución cada vez más cortos, se ha visto consolidarse una serie de métodos e instrumentos que ponen en evidencia el respaldo científico con el que cada vez más se han venido desarrollando numerosos estudios (Hendler, Shadbolt, Hall, Berners-Lee y Weitzner, 2008). Quizá con esta edición se haya podido mostrar parte de esa producción científica desde este lado del planeta.

Lo que queda claro y se puede afirmar con reserva a estas alturas es que las propuestas de estudio van en dos líneas: aquellas que entienden los fenómenos generados por la web como un constructo tecnológico (Hendler et al., 2008) y aquellas con un enfoque desde las humanidades y las ciencias sociales. Este último es visto desde los planteamientos teóricos de la ecología de medios que considera tanto los cambios a un nivel macro (social) en torno al ecosistema de medios como también los cambios a un nivel micro (individual) donde se analizan las audiencias en sus procesos cognitivos y perceptivos (McLuhan, 1962; Postman, 2000; Scolari, 2015).

Por lo demás, es interesante observar que desde estas dos perspectivas surgen marcos analíticos e interpretativos, así como técnicas y herramientas que pueden ir desde lo matemático cuantitativo hasta lo cualitativo, con claros enfoques multidisciplinarios. A esto, y a modo de subrayar su creciente importancia, se le suman distintos grupos de interés necesitados de conocimiento, insumo que la academia puede generar $\mathrm{y}$, de esta manera, retroalimentar a sectores que provienen de los ámbitos comerciales, políticos y gubernamentales, e incluso los educativos.

Entendamos, pues, que esta edición 28 de la revista Contratexto procura ubicar a "la práctica" como principal objeto de investigación, para abrir el diálogo a la comunidad académica y retroalimentar a todas las partes interesadas con un conocimiento basado en el estudio de los fenómenos que acondicionan y generan procesos 
disruptivos y transformaciones digitales. Así, se busca marcar el inicio de próximas compilaciones periódicas enfocadas en este tema de latente importancia.

Iniciamos nuestra sección Dosier con Lina Gómez, Alexandra Prieto y Ramón Borges (Puerto Rico), quienes presentan un análisis cuantitativo de 3000 mensajes en Twitter y ponderan la calidad de la información sobre la prevención en salud. Diana Oliveros (Colombia) aborda la categoría conceptual de capital TIC y propone una exploración cualitativa para comprender los vínculos entre capital cultural y prácticas tecnológicas. Diego Cerna (Perú) analiza la dinámica política cotidiana en los medios sociales para comprender la (re)producción de identidades políticas polarizadas, conocidas como el trol peruano. Silvia Gómez (Colombia) comparte su investigación sobre periodismo político digital y su comparación de formatos, contenidos y narrativas en tres medios digitales (Colombia, México y Brasil).

En nuestra sección Tendencias, contamos con tres aportes vinculados a otras áreas de la comunicación. Mauricio Coitiño, Rosario Queirolo y Alejandra Triñanes (Uruguay) comparan la cobertura periodística de la regulación del alcohol y de la marihuana, identificando los encuadres predominantes y el tratamiento diferenciado. Eugénie Richard y Álvaro Llano (Colombia) realizan un análisis semiótico de la comunicación de crisis en torno al proceso de pacificación en Colombia. Y, finalmente, Mathias Mäckelmann (Perú) reflexiona sobre la construcción de biografías presidenciales a partir del storytelling, examinando el caso del presidente Pedro Pablo Kuczynski.

Cierra nuestra edición la sección Reseñas sobre tres obras importantes para la comunicación social editadas este año. La primera reseña, a cargo de Eduardo Villanueva, trata sobre el libro Mecanismos de la posverdad de Jacqueline Fowks. La segunda reseña es presentada por Manuel Eyzaguirre sobre los dos volúmenes de Las miradas múltiples: cine regional peruano de Emilio Bustamante y Jaime Luna Victoria. La tercera reseña, por Julio César Mateus, trata sobre el trabajo Pedagogía red de Gros y Suárez-Guerrero.

Esperamos que todos los aportes de este número sean de especial utilidad para los estudiantes e investigadores de comunicaciones, humanidades y ciencias sociales. Cabe resaltar el esfuerzo académico de los autores y evaluadores, varios de los cuales colaboraron 
en medio de desastres naturales y conflictos políticos en sus países. Nuestra especial solidaridad con Venezuela, México y Puerto Rico.

Manuel Santillán Vásquez

Editor adjunto (sección Dosier)

Universidad de Lima

\section{Referencias}

Berners-Lee, T., Hall, W., Hendler, J., Shadbolt, N., y Weitzner, D. (2006). Creating a Science of the Web. Science, 313(5788), 769-771. doi: 10.1126/science.1126902

Hendler, J., Shadbolt, N., Hall, W., Berners-Lee, T., y Weitzner, D. (2008). Web Science: An Interdisciplinary Approach to Understanding the World Wide Web. Communications of the ACM, 51(7), 60-69. doi: 10.1145/1364782.1364798

McLuhan, M. (1962). The Gutenberg Galaxy. Toronto: University of Toronto Press.

Postman, N. (2000). The Humanism of Media Ecology. En Proceedings of the Media Ecology Association (pp. 10-16). Nueva York. Recuperado de http://w.media-ecology.org/publications/MEA_proceedings/v1/ postman01.pdf

Scolari, C. (2015). Ecología de los medios: entornos, evoluciones e interpretaciones. Barcelona: Gedisa. 